\title{
PERFIL PSICOLÓGICO DEL PACIENTE HIPERFRECUENTADOR EN UN CENTRO DE ATENCIÓN PRIMARIA PSYCHOLOGICAL PROFILE OF OVER-USES-ON-DEMAND PATIENTS IN A CENTER OF PRIMARY HEALTH CARE
}

\author{
FERnÁNDEZ-CADALSO, SANDRA \\ García-MartíneZ, JESÚs \\ Departamento de Personalidad, Evaluación y Tratamiento Psicológicos, \\ Facultad de Psicología, Universidad de Sevilla \\ jgm@us.es
}

Cómo referenciar este artículo/How to reference this article:

Fernández-Cadalso, S., y García-Martínez, J. (2012). Perfil psicológico del paciente hiperfrecuentador en un centro de atención primaria. Acción Psicológica, 9(2), 35-46 doi: http://dx.doi.org/10.5944/ ap.9.2.4102

\section{Resumen}

En este trabajo se presenta un estudio descriptivo del perfil psicológico del paciente hiperfrecuentador (PHF) en un centro de atención primaria mediante una aproximación constructivista. Se ha hecho énfasis tanto en los rasgos de personalidad como en el significado que estos sujetos hacen del fenómeno de la hiperfrecuentación. Para ello, se suministraron un cuestionario sociodemográfico ad hoc, el inventario de personalidad NEOFFI, y la técnica de la rejilla a un total de 30 pacientes multidemandantes. Aplicando un diseño descriptivo transversal, se encontró que existen asociaciones entre las distintas variables evaluadas y el neuroticismo, por lo que este puede ser considerado un buen predictor de la conducta hiperfrecuentadora. Los resultados de la rejilla indican que estos pacientes no están definidos por la presencia de dilemas, que se ven relativamente próximos a los demás y que hacen un mayor uso de constructos emocionales.

Palabras clave: hiperfrecuentación, atención primaria, personalidad, constructos personales.

\begin{abstract}
In this paper is presented a descriptive study about the psychological profile of over-using on demand patients in a center of primary health care. A constructivist approach is used in this study. The literature had remarked two variables as relevant ones in the study of over-using on-demand phenomenon, personality traits and meaning attribution. A sample of 30 over using patients was assessed using an ad-hoc socio-demographic inventory, the NE0-FFI Personality Inventory and a repertory grid. Applying a transversal exploration design, Neuroticism could be understood as a good predictor variable because it has relevant associations with a large number of the other studied variables. Repertory grid results show that over-using patients do not present dilemmas, see themselves similar to the others and use emotional constructs.
\end{abstract}

Keywords: over-using on-demand patients, primary health care, personality, personal constructs. 


\section{Introducción}

El fenómeno de la hiperfrecuentación está siendo objeto de estudio en un gran número de investigaciones en los últimos años por tratarse de un problema que tiene una gran incidencia en nuestro Sistema Público de Salud.

Se trata de usuarios y usuarias del sistema de salud que solicitan visitas médicas en porcentajes mucho mayores que el resto. Este problema genera varias consecuencias graves. Por un lado, un importante coste económico, debido al número de pruebas añadidas por los médicos para descartar patologías orgánicas; y por otro, el desgaste del personal sanitario provocado por la inespecificidad de los problemas que presentan estos pacientes.

Los llamados pacientes hiperfrecuentadores (PHF), son un pequeño grupo de usuarios del sistema sanitario (en torno al 3\%), pero que supone el 15\% del número total de visitas al médico de familia. Además, en términos generales, se estima que hacen un uso de entre el 21 y el 67\% de los recursos de atención primaria (Robles et al., 2009).

Es muy difícil establecer unos criterios a partir de los cuales podamos construir un perfil o una definición de estos pacientes. Las investigaciones que se han realizado hasta el momento han perseguido este objetivo unificador, pero ni las aproximaciones cualitativas ni las cuantitativas han logrado realizarlo de una forma homogénea y definitiva (Alameda y Pazos, 2009).

Son varios los factores desencadenantes que explican la multidemanda. Éstos se han agrupado en función de su relación con el individuo, con los profesionales sanitarios o con la estructura y funcionamiento del sistema de salud (Robles et al., 2009).

Los PHF responden a un perfil clínico determinado, siendo la atención primaria el primer nivel asistencial del sistema sanitario donde se tratan sus trastornos mentales más habituales (Aragonès, 2005). Suelen presentar: (a) enfermedades crónicas, (b) patologías psiquiátricas; y (c) problemas sociales.

En cuanto a los factores sociodemográficos y económicos, el perfil de los PHF es el siguiente (Alameda y Pazos, 2009; Llorente et al., 1996; Rodríguez, Cebrià, Corbella, Segura y Sobreques, 2003; Varela, 2000): son mujeres, aunque este hecho no es determinante; con edades comprendidas entre los 51 y los 59 años; amas de casa; tienen un bajo nivel de estudios; y pertenecen a una familia nuclear compuesta por padre, madre e hijos. También se ha asociado a la hiperfrecuentación al hecho de tener un bajo poder adquisitivo y un bajo nivel social (Karlsson, Joukamaa, Lahti, Lehtinen y Kokki-Saarinen, 1997; Savageu et al., 2006; cit. en Robles et al., 2009) y encontrarse desempleado (Borrell y Pasarín, 2004; cit. en García et al., 2007; Deaton y Paxson, 2001; Navarro y Benach, 1996; Sáez, 2003).

Los factores que hacen referencia a los profesionales sanitarios, señalan que los médicos se tienen que enfrentar a una problemática que va más allá de la simple lectura sintomática propia del modelo médico actual y del diagnóstico nosológico. Ante el desconocimiento de estos factores no puramente médicos, no pueden dar respuesta a la demanda del paciente, lo que acaba traduciéndose en una relación difícil entre éste y el profesional.

Por último, los factores relacionados con la estructura y la organización del Sistema de Salud son la accesibilidad al médico, la gratuidad de la asistencia y los trámites burocráticos, pero según apuntan Guerra y De Anca (2007) no son totalmente determinantes en lo que se refiere al hecho de acudir al centro de salud.

Los objetivos de este estudio son describir los rasgos de la personalidad de los pacientes hiperfrecuentadores, describir los constructos personales que habitualmente usan para construir su sistema de significados, analizar la estructura cognitiva y la construcción del sí mismo, estudiar las posibles correlaciones entre los rasgos y las variables de la rejilla y analizar las diferencias en función de la presencia o ausencia de dilemas.

\section{Método}

\section{Participantes}

Los participantes que componen la muestra son 30 sujetos hiperfrecuentadores: 7 hom- 
bres (19.4\%) y 23 mujeres (63.9\%), pacientes del Centro de Salud «Don Paulino García Donas» de Alcalá de Guadaira en la Provincia de Sevilla. Su media de visitas anual es de 18.17 $(\mathrm{SD}=6.417)$. Todos ellos presentan un diagnóstico difuso. Es decir, el elevado número de visitas que solicitan no está acorde con la gravedad de los problemas de salud que presentan ni con su tratamiento por lo que cabe la sospecha de que existan otros problemas subyacentes como serían los problemas psicosociales. El perfil sociodemográfico es bajo, siendo la mayoría amas de casa $(46.7 \%)$, con estudios primarios $(70 \%)$ y con unos ingresos económicos entre 1000 y 1500 euros.

\section{Instrumentos de Evaluación}

Con el fin de poder estudiar los objetivos anteriormente mencionados se han administrado a los sujetos de la muestra de hiperfrecuentadores un cuestionario de datos sociodemográficos, el Inventario de Personalidad NEO-FFI de Costa y McCrae (1999) y la Técnica de la Rejilla (Kelly, 1955) siguiendo el protocolo habitual del Proyecto Dilema (Feixas y Saúl, 2004).

En el dominio de los rasgos se utilizó la puntuación directa obtenida en cada uno de los cinco grandes factores del inventario NEO-FFI: neuroticismo $(\mathrm{N})$, extraversión $(\mathrm{E})$, apertura (O), amabilidad (A) y responsabilidad (C) (Costa y McCrae, 1999). Junto con este test se administró también la escala de distorsión del Cuestionario de los Cinco Grandes, que valora la tendencia a alterar las respuestas desde una perspectiva positiva (puntuaciones altas) hasta una negativa (puntuaciones bajas, Caprara, Barbanerelli y Borgogni, 1995).

La rejilla constaba de 12 elementos: Yo ahora, yo antes del problema, yo en el futuro, pareja, persona agradable, persona desagradable, médico, persona significativa 1 , persona significativa 2 , persona significativa 3 , persona significativa 4 y yo ideal.

El procedimiento de aplicación de la rejilla siguió los siguientes criterios:
(1). Los constructos se generaban a través de una comparación diádica. (2). El criterio de cese de la administración fue la obtención de una rejilla cuadrada (con tantos constructos como elementos). (3). Una vez finalizada la rejilla, todos los constructos se puntúan a través de todos los elementos utilizando una escala de intervalo de 7 puntos. Los valores del 1 al 3 servían para valorar la intensidad del polo de la izquierda, los valores del 5-7 para valorar la intensidad del polo de la derecha y el 4 indicaba la no aplicabilidad del constructo al elemento o la posición de equilibrio entre los dos polos. Así, en el constructo inteligente-perspicaz, 1 indica que la persona es muy inteligente, pero nada perspicaz, y 7 que es muy perspicaz, pero nada inteligente.

\section{Variables}

De la rejilla se utilizaron cuatro tipos de variables.

1. Las relativas al contenido de los constructos fueron el número de constructos en cada rejilla para las categorías moral, emocional, relacional, personal, operacional y valores, siguiendo los criterios de Feixas, Geldschläger y Neimeyer (2002).

2. Las variables relativas a la estructura del sistema de construcción fueron:

a) Construcciones funcionalmente independientes (FIC). Es un indicador del número de elementos y constructos que están agrupados entre sí e indica el nivel de complejidad de la construcción. Operativamente es el número de agrupaciones funcionales (dato generado por el programa Record en función de las correlaciones entre elementos y constructos), dividido por la suma total de elementos y constructos de la rejilla. En nuestro caso: x/24.

b) Porcentaje de Varianza Explicada por el Primer Eje (PVEF). Es el valor de la principal dimensión de significado, producto de un análisis de correspondencias simples previo. Cuanto mayor sea el valor de esa 
dimensión, menor es el número de componentes relevantes que se utilizan de la construcción de la experiencia. Teniendo en cuenta que los ejes representan dimensiones de significado, el valor de la varianza explicada del primer eje representa la magnitud de la principal dimensión de significado. Se considera el mejor indicador de la complejidad. Si el valor es alto, nos indica que el sujeto explica mucho acerca de su mundo en un solo eje, con lo cual podemos entender una cierta forma de construir su mundo "unidimensional». Si la puntuación fuera baja, estaríamos ante el caso de un sujeto con una mayor complejidad cognitiva (Feixas y Cornejo, 1996). En el caso del programa Record (Feixas y Cornejo, 2002), los datos se agrupan siempre en cinco ejes ordenados de mayor a menor en función del porcentaje de varianza explicada.

c) Índice de Bieri2. Es la capacidad para construir el sistema de significados de modo multidimensional. A mayor número de dimensiones, mayor es la capacidad para diferenciar la conducta de otros (Bieri et al., 1966). Según Feixas y Cornejo (1996), el índice de Bieri2, que utiliza la matriz reorganizada de datos y no los datos directos de la rejilla, es más adecuado que el índice de Bieri1 para medir la complejidad.

d) Intensidad. Es una medida del grado de integración de la construcción. Se trata de la suma del valor absoluto de las correlaciones de cada constructo con los demás, dividido por el total de constructos menos uno. A mayor valor, hay más integración entre constructos y la construcción es menos laxa (Bannister y Fransella, 1966).

e) Potencia Discriminativa. Es un índice que intenta ordenar jerárquicamente los constructos del sistema. Se calcula multiplicando el número de puntuaciones distintas por la diferencia entre la puntuación más alta y la más baja y dividiendo el producto por el número total de puntuaciones. Cuanto mayor es el valor, más jerarquizado está el sistema de construcción.

f) Polaridad Este índice muestra el grado en el que el sujeto utiliza puntuaciones extremas ( 1 o 7 ). Se calcula dividiendo el número de puntuaciones extremas entre el total de constructor. Las puntuaciones altas se consideran como una forma de rigidez cognitiva. Al contrario, las puntuaciones bajas nos dan una idea de la laxitud cognitiva del sujeto (Feixas y Cornejo, 1996).

g) Indefinición. Es el indicador opuesto a la polaridad. El porcentaje de valores no extremos usados por el sujeto. Indica la incapacidad para definir de forma de forma precisa la naturaleza de una construcción dada.

3. Variables relacionadas con la construcción del self. Se trata de correlaciones Pearson entre el yo actual, el yo ideal y el elemento-contenedor Otros. Otros es el resultado de la media de todos los elementos no relacionados con el yo (Feixas y Cornejo, 1996). Estas correlaciones permiten estudiar la autoestima del sujeto, siguiendo estos criterios:

a) Correlación yo actual-yo ideal: es una medida de la autoestima directa del sujeto, mide el grado de proximidad entre la autopercepción del sujeto y su imagen ideal.

b) Correlación yo actual-otros. Es el grado de proximidad entre la autopercepción y la percepción de los otros. Evalúa el grado de aislamiento social percibido (cuanto mayor sea el valor, menor es el aislamiento).

c) Correlación yo ideal-otros. Es el grado de proximidad entre la imagen ideal o meta personal y la percepción de los otros. Evalúa el grado en que otros se aproximan a la idealización, lo que implica el grado de adecuación atribuido a los demás. 
4. Dilema Implicativo. Una última variable considerada en nuestro análisis es la presencia del dilema implicativo. El dilema implicativo es la evidencia de una incoherencia en el sistema de construcción del sujeto, lo que supone que la persona va a tener dificultades en la elaboración y desarrollo de su conducta cuando para llevar a cabo una actividad tenga que utilizar simultáneamente los dos constructos implicados en el dilema.

Para entender la noción de dilema, hay que distinguir entre constructos congruentes y constructos discrepantes (Feixas y Saúl, 2004). Los primeros son aquellos en que la posición del yo actual coincide con la del yo ideal, de modo que la persona ubica ambos aspectos del yo en el mismo polo del constructo y con una intensidad similar. Los segundos son aquellos en que la posición del yo actual y el ideal diverge, de modo que el actual yo actual está ubicado en un polo distinto al yo ideal. Matemáticamente se define el constructo discrepante como aquel en que la diferencia entre el valor otorgado al yo ideal y al yo actual es al menos de cuatro puntos absolutos (por ejemplo, en el constructo inteligentehábil, si el yo actual tuviera un valor de 2 , pero el yo ideal de 7 , se obtendría la siguiente diferencia, $2-7=-5$. El valor 5 indicaría que este constructo es discrepante para el sujeto).

El dilema implicativo se produce cuando se activan simultáneamente un constructo congruente y otro discrepante y además estos están correlacionados positivamente entre sí, de manera que un cambio en un constructo (un cambio de ubicación del yo actual entre los polos) constructo) conlleva un cambio en el otro (también un cambio de ubicación del yo actual entre los polos).

\section{Procedimiento}

\section{Reclutamiento de los sujetos}

Previamente al inicio de la investigación, se acuerdan con los médicos de familia que cola- boran en el estudio las características principales que deben cumplir los sujetos que formaran parte de la muestra. Una vez realizada esta primera fase de aclaración de criterios, se procede a la selección al azar de los sujetos mediante la Base de Datos de Usuarios (BDU) del Servicio Andaluz de Salud teniendo en cuenta el criterio de inclusión referente al número de visitas.

\section{Recogida de la información}

Con el reclutamiento de los participantes ya finalizado, se confeccionó una carta en la que se invitó al paciente a participar en el estudio. Tras el envío de la misma a cada uno de los sujetos, se dio un margen de una semana a partir del cual la investigadora se puso en contacto con cada uno de ellos vía telefónica y se da inicio al proceso de citación.

\section{Tipo de diseño}

Se trata de un estudio transversal ex post facto. Las variables dependientes son el tipo de sujetos (totalidad de la muestra o sujetos consin dilemas, en función del análisis) y las variables dependientes son las distintas medidas del NEO-FFI y de la rejilla.

\section{Análisis de datos}

Como esta investigación pretende seguir un diseño básicamente descriptivo, se ha usado una estadística exploratoria que ha permitido describir y analizar la información recogida en los cuestionarios descritos anteriormente para la muestra de pacientes hiperfrecuentadores. Si bien en algunos casos se han usado técnicas inferenciales como el coeficiente de correlación de Pearson y el análisis de la varianza para analizar la posible existencia de asociaciones entre las distintas variables estudiadas. Todas las variables han sido codificadas para establecer un formato cuantitativo, incluso en variables cualitativas o de tipo nominal. 


\section{Resultados}

\section{Rasgos de la personalidad}

Como se puede observar en figura 1 que se muestra a continuación, desde el punto de vista de los rasgos de personalidad, los hiperfrecuentadores se definen por el neuroticismo. Por tanto, se pone de manifiesto la relevancia que tiene éste como predictor de la problemática psicológica en los pacientes hiperfrecuentadores.

Se ha considerado también relevante destacar otras puntuaciones obtenidas en el cuestionario de personalidad NEO-FFI así como la puntuación media-alta en la escala de "extraversión» y las bajas puntuaciones en las dimensiones «apertura» $\mathrm{y}$ «responsabilidad».

\section{Descripción del contenido de los constructos utilizados}

El análisis del contenido de los constructos se ha hecho siguiendo el sistema de clasifica- ción de constructos personales propuesto por Feixas et al. (2002). La adscripción de categorías se hizo mediante un procedimiento interjueces, realizándose independientemente por los autores del trabajo. La correlación interjueces fue de 0,78. Los constructos del área emocional son los más usados por los pacientes hiperfrecuentadores con una media de 3,86.

\section{Análisis de la Estructura cognitiva y de la construcción del sí mismo}

\section{Estructura cognitiva}

La media de la intensidad resulta ser 0.27 , lo cual indica de que los pacientes hiperfrecuentadores tienen una estructura cognitiva poco intensa y poco integrada. Por tanto, los constructos generados son poco discriminativos.

La potencia es de 1.56, lo que supone una baja integración jerárquica del sistema. Es decir, existe muy poca flexibilidad o sutilidad al aplicar los constructos. Se trata de personas poco creativas.

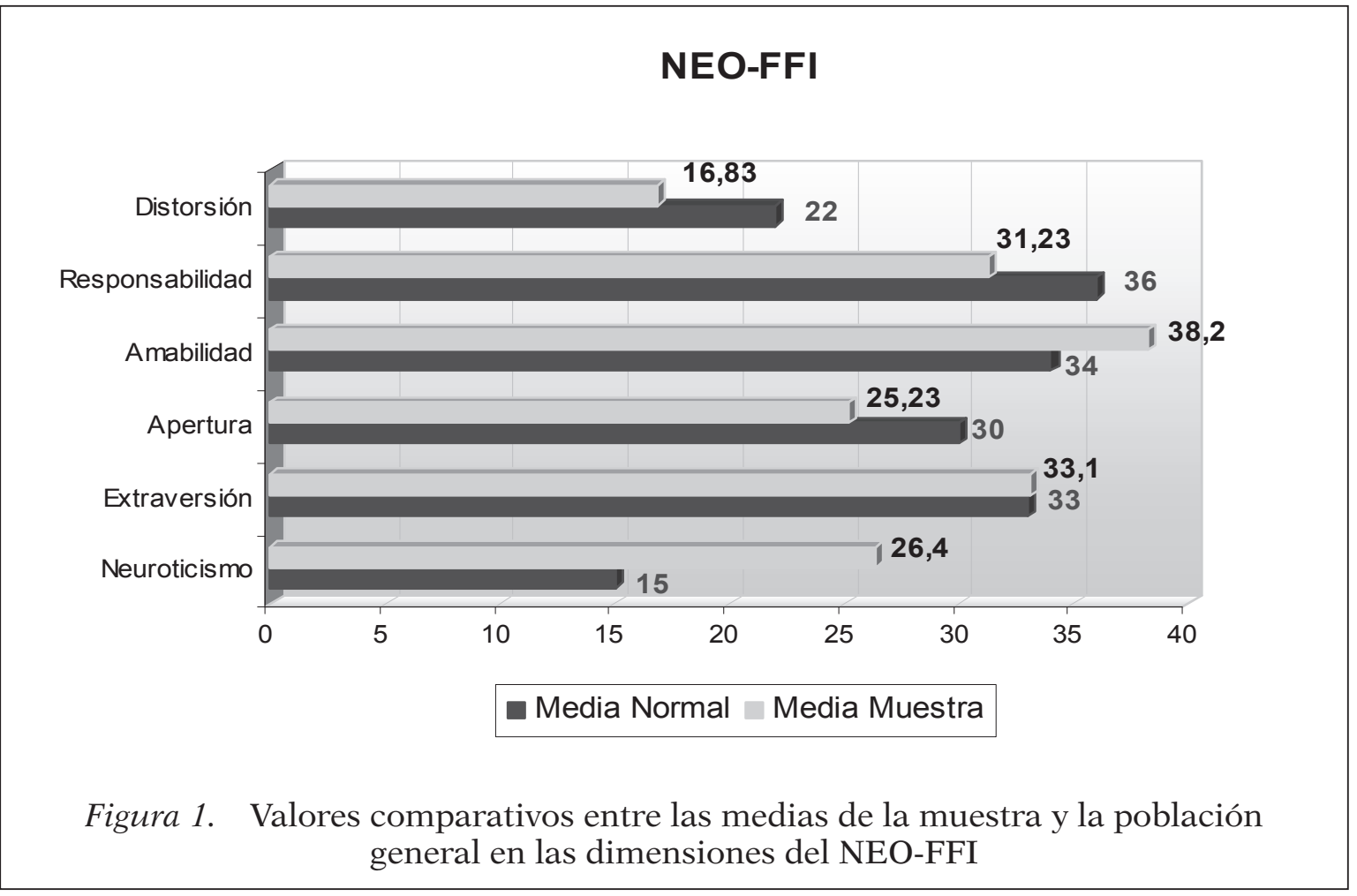


La polarización, con una media de 58.30, refleja que los sujetos tienden a dar puntuaciones extremas, lo que significa que existe un cierto grado de rigidez cognitiva.

La indefinición es de 9.2, es decir, un valor muy bajo. Por tanto, la mayoría de los sujetos hiperfrecuentadores han sido capaces de situar los distintos elementos de la rejilla en un polo u otro del constructo, dándoles sentido de forma significativa.

El número de constructos funcionalmente independientes (FIC) total es de 7.92 sobre 24. Es, por tanto, una puntuación baja que indica que los sujetos están usando pocos constructos claramente diferenciables entre sí.

El índice de Bieri2, con un valor de 0.41 aporta información acerca de la complejidad cognitiva. Al ser un valor que no es bajo, no podemos afirmar que haya una tendencia clara a la simplicidad o la complejidad.

La varianza explicada por el primer eje es del $12 \%$, lo que supone que existen otros factores que igualmente intervienen en la construcción del mundo interpersonal de los sujetos. Este resultado es indicador de una relativa complejidad del sistema, dado que son necesarios varios ejes para interpretar el sistema de construcción de las hiperfrecuentadores. Dado que el índice de Bieri2 también se orienta más a la complejidad que a la simplicidad, es probable que el sistema de los hiperfrecuentadores sea relativamente complejo, pero es posible que no esté bien integrado. La complejidad también se ve reflejada en el valor medio de la intensidad de los dilemas (0.46).

El 47\% de la muestra presenta dilemas. Por tanto, no se puede considerar a los sujetos hiperfrecuentadores como especialmente dilemáticos.

\section{Construcción del sí mismo}

La correlación entre el «yo»y el «yo ideal» es de 0.32 , lo que refleja que los pacientes hiperfrecuentadores tienden en general a verse relativamente próximos a su ideal. La correlación entre el «yo»y los «otros» es de 0.43. Esta puntuación nos indica que se construyen de un modo bastante semejante a los demás. La correlación entre el "yo ideal» y los «otros» es la más elevada de las tres, con una media de 0.57. Esto indica que ven a las personas que les rodean cerca de su ideal personal.

Este conjunto de correlaciones corresponde con un perfil general de positividad y adecuación, no pudiéndose detectar problemas generales de autoestima comparativa (Feixas y Cornejo, 1996).

\section{Correlaciones entre indicadores estructurales de la rejilla y rasgos}

En cuanto a las correlaciones ligadas a los indicadores de la rejilla, cabe señalar que la intensidad correlaciona significativamente con el FIC ( $\mathrm{r}=-0.475, p=0.011)$, por lo que los sujetos más complejos tienden a usar menos constructos de forma independiente. La intensidad también correlaciona significativamente con el índice Bieri2 ( $\mathrm{r}=0.400, p=0.35)$ lo que refleja que los sujetos más complejos construyen los acontecimientos desde varios puntos de vista. Y por último, la intensidad correlaciona significativamente con la varianza explicada por el primer eje $(r=0.722, p=0.0001)$ de modo que los sujetos más complejos construyen su mundo interpersonal de forma unidimensional.

La potencia ha correlacionado significativamente con el rasgo responsabilidad $(r=-0.463$, $p=0.013$ ), por lo que los sujetos responsables tienden a ser personas poco creativas o sutiles. También ha correlacionado significativamente con la distorsión ( $r=0.436, p=0.20$ ) lo que refleja que quienes son más sutiles, falsean en menor grado sus respuestas en las diferentes pruebas. La polarización ha obtenido una correlación significativa con la potencia $(r=-0.620$, $p=0.0001$ ), de manera que aquellos sujetos más creativos son menos rígidos cognitivamente. La correlación entre el número de constructos funcionalmente independientes y la potencia también es significativa $(r=0.560$, $p=0.002$ ), lo que informa de que los sujetos más creativos tienden a usar más constructos de forma independiente. Además, por lo que se 
observa en la correlación entre potencia y el índice de Bieri2 ( $\mathrm{r}=-0.743, p=0.0001)$, cuanta mayor sutilidad, menor complejidad cognitiva tienen los sujetos. Y por último, señalar la correlación significativa que se establece entre la correlación Yo-Ideal y la potencia $(r=0.434$, $p=0.15$ ), lo que significa que aquellas personas que se ven más cerca de su ideal tienden a ser menos sutiles en su formato de construcción.

La polarización ha correlacionado significativamente con la amabilidad ( $\mathrm{r}=0.462, p=0.13)$, por lo que los sujetos más amables tienen una tendencia a ser más rígidos cognitivamente. Y por último, existen correlaciones significativas entre polarización y FIC $(\mathrm{r}=0.551, p=0.002$, índice de Bieri2 ( $\mathrm{r}=0.836, p=0.0001)$ y PVEPF $(r=0.402, p=0.34)$. Por tanto, los sujetos más rígidos usan menos constructos de forma independiente y tienden a construir su mundo interpersonal de forma unidimensional.

En cuanto al índice de indefinición, se han encontrado correlaciones significativas con la amabilidad $(\mathrm{r}=-0.410, p=0.030$ y la responsabilidad ( $r=-0.383, p=0.044)$, por lo que los sujetos más amables y responsables tienden a tener menor dificultad para dar sentido a los elementos de una forma significativa. Por lo contrario, la correlación significativa que existe entre indefinición y FIC ( $\mathrm{r}=0.741, p=0.0001)$, indica que quienes usan más constructos de forma independiente, tienen mayor dificultad para situar los elementos en un polo $u$ otro de los constructos. Por último, las correlaciones significativas que se observan entre la indefinición, el índice de Bieri2 ( $\mathrm{r}=-0.375, p=0.049)$ y la frecuencia de constructos con contenidos personales $(r=-0.432, p=0.022)$ indican que los sujetos con mayor índice de indefinición son menos complejos y usan menos constructos referidos a la naturaleza de las personas. El FIC ha correlacionado significativamente con la responsabilidad ( $\mathrm{r}=-0.507, p=0.006)$ y con el índice de Bieri2 ( $\mathrm{r}=-0.581, p=0.01)$, lo que refleja que los sujetos que usan diversos constructos de forma independiente son menos responsables y también menos complejos. No obstante, usan más constructos emocionales $(\mathrm{r}=0.454, p=0.015)$.
El índice de Bieri2 ha correlacionado significativamente con la amabilidad $(r=0.413$, $p=0.029)$, la distorsión $(\mathrm{r}=0.434, p=0.021) \mathrm{y}$ la PVEPF ( $\mathrm{r}=0.404, p=0.033)$. Estos resultados indican que los sujetos más complejos suelen ser más amables, también tienden a sesgar las respuestas de las pruebas y construyen su mundo interpersonal de forma unidimensional.

La correlación Yo-Ideal ha obtenido correlaciones significativas con la correlación IdealOtros ( $\mathrm{r}=0.384, p=0.043$ ), por lo que los sujetos que tienden a verse cerca de su ideal suelen ver también a los otros cerca de su ideal.

La correlación Yo-Otros ha correlacionado significativamente con el uso de constructos morales $(r=-0.390, p=0.040)$. Por tanto, los sujetos que se ven más próximos a los otros, no usan constructos morales.

En cuanto a la correlación Ideal-Otros, existe una correlación significativa con la responsabilidad $(\mathrm{r}=-0.385, p=0.043)$, la correlación Yo-Ideal $(\mathrm{r}=0.384, p=0.043)$ y con los constructos morales $(\mathrm{r}=0.532, p=0.004)$. Con lo cual, aquellos sujetos que tienden a ver a los demás cerca de su ideal son menos responsables, se ven próximos a su ideal y suelen hacer uso de los constructos morales.

Por último, se ha observado una correlación significativa entre PVEPF y la frecuencia de constructos emocionales ( $\mathrm{r}=-0.399, p=0.037$ ) que indica que los sujetos que presentan un grado de unidimensionalidad al construir su mundo interpersonal, hacen un uso menor de los constructos emocionales.

\section{Diferencias en función de la presencia o ausencia de dilemas en la muestra de pacientes hiperfrecuentadores}

Para comprobar si la presencia o ausencia de dilemas genera alguna diferencia en la muestra de hiperfrecuentadores en relación con el resto variables del estudio, se procedió a un análisis intersujetos. Dado el escaso tamaño de las submuestras (15 sujetos sin dilemas, 13 con dilemas) se optó por utilizar estadísticos no paramétricos, en concreto la prueba U de Mann-Whit- 
ney para establecer estos contrastes, usando como valor de probabilidad el correspondiente a la significación asintótica bilateral.

No se encuentran diferencias significativas para ninguno de los rasgos de personalidad, por lo que se puede decir que la estructura de rasgos es semejante para hiperfrecuentadores con y sin dilemas. En cuanto a los indicadores de la rejilla, sólo aparece una diferencia significativa para la correlación yo-ideal $(\mathrm{U}=44$; valor $\mathrm{Z}=-2.464$, $\mathrm{p}$ asintótica bilateral $=0.014$ ). Dado que el rango promedio del grupo sin dilemas es 18.07 y el del grupo con dilemas 10.30, se puede afirmar que el grupo sin dilemas tiene una visión de sí mismos más adecuada a su ideal.

\section{Discusión}

Son muy pocos los estudios que se han centrado en indagar acerca de los aspectos psicológicos de los pacientes hiperfrecuentadores. La mayoría de las investigaciones publicadas hasta el momento hacen referencia a los factores que provocan el fenómeno de la hiperfrecuentación (del paciente, del médico y de la organización), así como las consecuencias que este fenómeno provoca en el ámbito de la salud.

Tal y como aparece en la mayoría de estudios sociodemográficos (Llorente et al., 1996; Rodríguez et al., 2003; Varela, 2000), que tratan el fenómeno de la hiperfrecuentación, se trata de un fenómeno que afecta en su gran mayoría a mujeres, frente a un pequeño porcentaje de hombres. Los hiperfrecuentadores se caracterizan por pertenecer a un sistema familiar compuesto por padres e hijos, aunque en algunos casos también forma parte del sistema familiar algún otro pariente directo. En cuanto al nivel académico, un gran porcentaje de los sujetos refiere haber cursado tan sólo los estudios primarios, por lo que es bastante bajo. Esto concuerda con el tipo de profesiones a las que se suelen dedicar los pacientes hiperfrecuentadores, pues gran parte de ellos desempeña tareas domésticas u otro tipo de trabajos que requieren poca formación. El escaso nivel de ingresos económicos a nivel fa- miliar también es un aspecto en el que se coincide con otros trabajos (Karlsson, Joukamaa, Lahti, Letinen y Kokki-Saarinen, 1997; Savageu et al., 2006).

Otro de los aspectos que caracteriza a los pacientes hiperfrecuentadores es el elevado número de visitas anuales que realizan, se puede decir que la media obtenida que es de 18.17 es coherente con lo que se ha publicado hasta el momento (Guerra y De Anca, 2007).

En cuanto a los resultados obtenidos acerca de los rasgos de personalidad, estos sujetos responden a un perfil básicamente neurótico resultado que concuerda con los estudios realizados por Rodríguez et al. (2003) y Kroenke (2007), quienes ya apuntaron la relación entre sintomatología ansiosa-depresiva e hiperfrecuentación.

Sobre la manera como se construyen a sí mismos, los sujetos hiperfrecuentadores no se ven lejanos a su ideal, pero sí diferentes a los demás. Los valores de la rejilla aportan datos sobre la estructura cognitiva de los sujetos y de cómo se construyen a sí mismos. Así pues, acorde con los resultados obtenidos, se puede indicar que los pacientes hiperfrecuentadores son poco creativos, tienen una cierta rigidez cognitiva. Los distintos indicadores de complejidad no son coherentes entre sí, aunque es probable que más que baja complejidad tengan problemas de integración de los constructos. En cualquier caso, el porcentaje de varianza explicado por el primer factor no puede usarse como un indicador fiable de complejidad con estas muestras.

Los resultados indican también que los pacientes hiperfrecuentadores usan pocos tipos de constructos y que los más habituales son los que pertenecen a las áreas emocional y moral, resultado que coincide con el aportado por Montesano, Feixas, y Varlotta (2009) en un estudio realizado a un grupo de personas con depresión con el objetivo de analizar los constructos personales más usados por este colectivo.

Para finalizar, este estudio muestra la gran relevancia de los factores psicológicos en el fenómeno de la hiperfrecuentación y por tanto 
pone de manifiesto la necesidad de que los profesionales de la salud abandonen el modelo lineal «síntoma-diagnóstico-tratamiento» que vienen empleando hasta el momento, para empezar a enfrentarse a los problemas psicosomáticos mediante un enfoque global en el que tengan en cuenta otros factores como los sociales y psicológicos.

Entre las limitaciones de este estudio cabe señalar el pequeño tamaño de la muestra y la falta de una muestra control comparativa de sujetos normofrecuentadores o que padezcan una enfermedad crónica o un estado médico asimilable a la hiperfrecuentación en términos de la indefinición del síndrome para poder obtener diferentes perfiles entre muestras clínicamente contrastables.

\section{Conclusiones}

En resumen, las conclusiones de este estudio son las siguientes:

- Los sujetos hiperfrecuentadores tienen tendencia al neuroticismo y éste puede ser un buen predictor de la conducta hiperfrecuentadora.

- En cuanto a su estructura cognitiva, no se puede afirmar que sean sujetos simples, si bien tampoco son claramente complejos. Si presentan una alta polaridad.

- No se puede afirmar que los sujetos hiperfrecuentadores estén definidos por la presencia de dilemas. Tampoco existen diferencias significativas entre los rasgos de la personalidad y la presencia de dilemas. 


\section{Referencias}

Alameda, A., y Pazos, A. (2009). Investigación cualitativa sobre la conceptualización de la hiperfrecuentación por parte del personal médico de atención primaria [Qualitative Research on the Conceptualization around the Frequent Attendance by Primary Medical Staff]. Revista Española de Salud Pública, 83(6), 863-875.

Aragonès, E. (2005). Estudio de los trastornos depresivos en la atención primaria de salud [Study of depressive disorders in primary health care]. Revista de psiquiatría de la facultad de medicina de Barcelona, 32(1), 30-37.

Bannister, D., y Fransella, F. (1966). A grid test of schizophrenic thought disorder. British Journal of Social and Clinical Psychology, 5, 95-102.

Bieri, J., Atkins, A. L., Briar, S., Leadman, R. L., Miller, H., y Tripodi, T. (1966). Clinical and social judgment: the discrimination of behavioural information. New York: Wiley.

Borrell, C., y Pasarín, M. I. (2004). Desigualdad en salud y territorio urbano [Inequalities in health and urban areas]. Gaceta Sanitaria, 18, 1-4.

Caprara, G., Barbaranelli, C., y Borgongi, L. (1995). Big Five Questionnaire (adaptación española de J. Bermúdez). Madrid, España: TEA.

Costa, P. T., y McCrae, R. R. (1999). Inventario de personalidad NEO revisado (NEO PI-R) / Inventario $N E O$ reducido de cinco factores (NEO-FFI): manual profesional. Madrid, España: TEA.

Deaton, A., y Paxson, C. (2001). Mortality, education, income and inequality among American Cohorts. Cambridge: National Bureau of Economic Research. NBER Working Paper: 7140.

Feixas, G., y Cornejo, J. M. (1996). Manual de la técnica de rejilla mediante el programa RECORD ver 2.0. [Grid Tecnique Manual using the Gricord Soft- ware]. Barcelona, España: Paidós.

Feixas, G.; Geldschläger, H y Neimeyer, R. A. (2002). Content Analysis of Personal Constructs. Journal of Constructivist Psychology, 15, 1-19.

Feixas, G., y Saúl, L. A. (2004). The multi-center dilemma project: An investigation on the role of cognitive conflicts in health. The Spanish Journal of Psychology, 7, 69-78.
García, M. A., Martín, V., Ramírez, D., González, Y., Hidalgo, I., Escribano, E.,... y De la Cámara, A. (2007). Factores socioeconómicos y frecuentación en las consultas de medicina de familia de la red sanitaria pública madrileña [Socioeconomic factors and utilization of public family practice facilities in Madrid]. Gaceta Sanitaria, 21(3), 219-26.

Guerra, J. A., y De Anca, I. A. (2007). Motivos que condicionan el uso de los servicios sanitarios por parte de los pacientes hiperfrecuentadores: estudio con grupos focales [Motives That Condition Use of the Health Services by Over-Users: Study With Focus Groups]. Atención Primaria, 39(7), 349-54.

Karlsson, H., Joukamaa, M., Lahti I., Letinen, V., y Kokki-Saarinen, T. (1997). Frequent attender profiles: different clinical subgroups among frequent attenders patients in primary care. Journal of Psychosomatic Research, 42, 157-66.

Kroenke, K. (2007). Efficacy of Treatment for Somatoform Disorders: A review of Randomized Controlled Trials. Psichosomatic Medicine, 69, 881-888.

Llorente, S., López, T., García, L. J., Alonsa, M., Alonso, P., y Muñoz, P. (1996). Perfil del hiperfrecuentador de un centro de salud [Profile of the health centre's over-user]. Atención Primaria, $17,20-26$.

Montesano, A., Feixas, G., y Varlotta, N. (2009). Análisis de contenido de constructos personales en la depresión [Content analysis of personal constructs in depression]. Salud Mental, 32, 371-379.

Navarro, V., y Benach, J., y la Comisión científica de estudios de las desigualdades sociales en salud en España (1996). Desigualdades sociales en salud en España [título en inglés]. Madrid, España: Ministerio de Sanidad y Consumo y The School of Hygiene and Public Health. The Johns Hopkins University.

Robles, R., Gili, M., Gelabert, J., Llull, M., Bestard, F., Vicens, C.,... y Roca, M. (2009). Características sociodemográficas y psicopatológicas del paciente hiperfrecuentador en atención primaria [título en inglés]. Actas Españolas de Psiquiatría, 37(6), 320-325.

Rodríguez, C., Cebrià, J., Corbella, S., Segura, J., y Sobreques, J. (2003). Rasgos de personalidad y malestar psíquico asociados a los pacientes hi- 
perfrecuentadores de consultas de Atención Primaria [Social inequalities in health in Spain]. Revista de Medicina Familiar y Comunitaria, 13(3), 143-150.

Sáez, M. (2003). Condicionantes en la utilización de los servicios de atención primaria. Evidencias empíricas e inconsistencias metodológicas [Factors conditioning primary care services utilization. Empirical evidence and methodological inconsistencies]. Gaceta Sanitaria, 18, 1-4.
Savageu, J. A., McLoughlin, M, Ursan, A., Bai Y., Collins, M., y Cashman, S. M. (2006). Characteristics of frequent attenders at a community health center. Journal of the American Board of Family Medicine, 19, 265-75.

Varela, F. J. (2000). Disfunción familiar en hiperfrecuentadores de atención primaria [Family dysfunction and frequent use or primary care services]. Revista Medicina de Familia (Andalucía), 1(2), 141-144. 